\title{
Pseudoscalar Decays into Two Photons in Chiral Perturbation Theory
}

\author{
Johan Bijnens \\ Sektion Physik, Universität München, Theresienstrasse 37, D-8000 Munich 2, Federal Republic of Germany
}

Albert Bramon

Departament de Física, Universitat Autonoma de Barcelona, 08193 Bellaterra, Barcelona, Spain

and

Fernando Cornet

Max-Planck-Institut für Physik und Astrophysik, Werner-Heisenberg-Institut für Physik, P.O. Box 401212 , Munich, Federal Republic of Germany

(Received 29 June 1988)

\begin{abstract}
We study the one-loop corrections in chiral perturbation theory to the $\gamma \gamma$ and $\gamma \gamma^{*}$ decays of the pseudoscalar mesons. The latter are divergent, thus requiring the existence of dimension-six terms contributing to the anomalous Lagrangian. Some examples of such terms are given. We also discuss the experimental consequences of the next-to-leading terms.
\end{abstract}

PACS numbers: $13.40 . \mathrm{Hq}, 11.40 . \mathrm{Fy}$

The Langrangian of QCD is known to have a $\mathrm{U}(3)_{L}$ $\otimes U(3)_{R}$ chiral symmetry when the three lightest quark masses are set to zero. This symmetry is believed to be spontaneously broken to its vector $\mathrm{U}(3)_{L+R}$ subgroup via nonvanishing quark vacuum expectation values $\langle\bar{q} q\rangle_{0}$. The nonet of (pseudoscalar) Goldstone bosons associated with the spontaneous breaking of the symmetry can be conveniently parametrized in terms of

$$
\Sigma=\exp (2 i M / f)
$$

with $M=\pi^{a} \lambda^{a} / \sqrt{2}+\eta_{1} 1 / \sqrt{3}$ and transforming under $\mathrm{U}(3)_{L} \otimes \mathrm{U}(3)_{R}$ as $\Sigma \rightarrow \Sigma^{\prime}=L \Sigma R^{\dagger}$. The corresponding Lagrangian, to lowest order in derivatives and mass terms (dimension two), is given by

$\mathcal{L}_{2}=\frac{f^{2}}{8} \operatorname{tr}\left(D_{\mu} \Sigma D^{\mu} \Sigma^{\dagger}\right)+v \operatorname{tr}\left(m \Sigma+m \Sigma^{\dagger}\right)-\frac{4}{3 f^{2}} v^{\prime} \eta_{1}^{2}$,

where the first term provides the kinetic energy for the mesons and interaction terms consistent with current algebra and the second one describes the explicit breaking of $\mathrm{U}(3)_{L} \otimes \mathrm{U}(3)_{R}$ by the quark masses. The effect of the breaking of $\mathrm{U}(1)_{A}$ through quantum loops (instanton effects) is included only via an extra mass term for $\eta_{1}$. The covariant derivative is $D_{\mu} \Sigma=\partial_{\mu} \Sigma+i e[Q$, $\Sigma$ ] $A_{\mu}$, where $A_{\mu}$ is the photon field, and the quark mass matrix $m$ and quark charge matrix (in units of $e$ ), $Q$, are given by $m=\operatorname{diag}\left(m_{u}, m_{d}, m_{s}\right)$ and $Q=\operatorname{diag}\left(\frac{2}{3},-\frac{1}{3}\right.$, $-\frac{1}{3}$ ). The Lagrangian $\mathcal{L}_{2}$, Eq. (2), describes the lowest-order strong and electromagnetic interactions of the pseudoscalar-meson nonet.

Chiral perturbation theory ${ }^{1,2}$ starts with the Lagrangian $\mathcal{L}_{2}$ and proceeds to a higher-order expansion in terms of momenta and quark masses. The resulting effective low-energy theory is completely known to nextto-leading order, once the $\eta_{1}$ has been integrated out. ${ }^{2}$ It contains two distinct types of terms. The first ones are originated by pseudoscalar loops with vertices deducible from $\mathcal{L}_{2}$ giving rise to analytic and nonanalytic contributions. The latter are particularly interesting, since they are considered to be the dominant ones and, on the other hand, involve a dependence on the renormalization scale $\mu$. As a result of this, the second type of (counter)terms, eliminating that $\mu$ dependence and the associated divergences, is required. The set of all the counterterms appearing in the next-order Lagrangian, $\mathcal{L}_{4}$, has been identified and extensively analyzed by Gasser and Leutwyler. $^{2}$

The problem of treating anomalies in an effective low-energy theory was solved long ago by Wess and $\mathrm{Zu}$ mino $^{3}$ and has been elegantly reformulated by Witten. ${ }^{4}$ The preceding nonanomalous Lagrangians have to be implemented by anomalous terms. To lowest order these extra terms in the effective Lagrangian are

$$
\begin{aligned}
\mathcal{L}_{4}^{\text {anom }}= & i\left(e^{2} / 8 \pi^{2}\right) \epsilon^{\mu v \alpha \beta}\left(\partial_{\mu} A_{v}\right) A_{\alpha} \\
& \times \operatorname{tr}\left(Q^{2} \partial_{\beta} \Sigma \Sigma^{\dagger}+Q^{2} \Sigma^{\dagger} \partial_{\beta} \Sigma+\frac{1}{2} Q \Sigma Q \Sigma^{\dagger} \partial_{\beta} \Sigma \Sigma^{\dagger}-\frac{1}{2} Q \Sigma^{\dagger} Q \Sigma \partial_{\beta} \Sigma^{\dagger} \Sigma\right)-i\left(e / 16 \pi^{2}\right) \epsilon^{\mu v \alpha \beta} A_{\mu} \\
& \times \operatorname{tr}\left[Q\left(\partial_{v} \Sigma \Sigma^{\dagger}\right)\left(\partial_{\alpha} \Sigma \Sigma^{\dagger}\right)\left(\partial_{\beta} \Sigma \Sigma^{\dagger}\right)+Q\left(\Sigma^{\dagger} \partial_{v} \Sigma\right)\left(\Sigma^{\dagger} \partial_{\alpha} \Sigma\right)\left(\Sigma^{\dagger} \partial_{\beta} \Sigma\right)\right]+\cdots,
\end{aligned}
$$

where the dots refer to nonphotonic terms. As in the previously discussed nonanomalous part, three types of contributions are expected to appear at next order in the chiral perturbation expansion: (i) loop diagrams involving one vertex 
from $\mathcal{L}_{4}^{\text {anom }}$ and the other vertices from $\mathcal{L}_{2}$, Eq. (2); (ii) tree diagrams involving one vertex from $\mathcal{L}_{4}^{\text {anom }}$, one from $\mathcal{L}_{4}$ and any number of vertices from $\mathcal{L}_{2}$ (these diagrams, however, do not contribute to the processes we study in this Letter); and (iii) tree diagrams from a dimension-six anomalous Lagrangian $\mathcal{L}_{6}^{\text {anom }}$. The coefficients in $\mathcal{L}_{6}^{\text {anom }}$ can be used to absorb possible divergences appearing in the calculation from contributions (i) and (ii). Some examples of terms of $\mathcal{L}_{6}^{\text {anom }}$ are given in Ref. 5. In this paper we want to study their contribution to the two-

$$
\begin{aligned}
& f_{\pi}=f\left(1-2 \mu_{\pi}-\mu_{K}+\frac{64 v}{f^{4}}\left[\left(2 m_{u, d}+m_{s}\right) L_{4}^{r}+m_{u, d} L_{5}^{r}\right]\right), \\
& f_{K}=f\left[1-\frac{3}{4} \mu_{\pi}-\frac{3}{2} \mu_{K}-\frac{3}{4} \mu_{\eta_{8}}+\frac{64 v}{f^{4}}\left[\left(2 m_{u, d}+m_{s}\right) L_{4}^{r}+\frac{m_{u, d}+m_{s}}{2} L_{5}^{r}\right)\right], \\
& f_{\eta_{8}}=f\left[1-3 \mu_{K}+\frac{64 v}{f^{4}}\left[\left(2 m_{u, d}+m_{s}\right) L_{4}^{r}+\frac{m_{u, d}+2 m_{s}}{3} L_{5}^{r}\right)\right], \\
& f_{\eta_{1}}=f\left[1+\frac{64 v}{f^{4}}\left(\left(2 m_{u, d}+m_{s}\right) L_{4}^{r}+\frac{2 m_{u, d}+m_{s}}{3} L_{5}^{r}\right)+\cdots\right],
\end{aligned}
$$

where the simplicity of the last line (concerning the loop part) is due to the SU(3) singlet nature of $\eta_{1}$ and the dots represent unknown, higher-dimension terms contributing only to $\eta_{1}$. $L_{4}^{r}$ and $L_{5}^{r}$ are the renormalized coupling constants of the two relevant terms of the Lagrangian $\mathcal{L}_{4 .}{ }^{2}$ There, the first three equations of (5) can also be found and the value of $L_{4}^{r}$ has been argued to be consistent with zero. The experimental value ${ }^{6} f_{K} / f_{\pi}=1.22$ \pm 0.01 allows then to estimate the constant $L_{5}^{r}$. At $\mu$ $=m_{\eta}=0.55 \mathrm{GeV}$, the contribution of the chiral logarithms to $f_{K} / f_{\pi}$ is negligible and one obtains $L_{5}^{r}=(2.2$ $\pm 0.5) \times 10^{-3} .^{2}$ Using these values for $L_{4}^{r}$ and $L_{5}^{r}$ and neglecting the extra counterterms we get $f_{\eta_{1}} / f_{\pi} \simeq 1.1$. Alternatively, if we choose $\mu \sim 1.5 \mathrm{GeV}$, where $f_{K} / f_{\pi}$ is described by the chiral logarithms $\left(L_{5}^{r}=0\right)$, we obtain $f_{\eta_{1}} / f_{\pi} \sim 0.9$, still neglecting the extra counterterms. In any case, the absolute correction in $f_{\pi}$ is around $10 \%$. Therefore, we will hereafter adopt $f_{\eta_{8}} / f_{\pi}=1.3$ and leave $f_{\eta_{1}} / f_{\pi}$ free.

We now turn to the $P \rightarrow \gamma \gamma$ decays following the photon decays of the neutral pseudoscalar mesons.

The decay widths $P \longrightarrow \gamma \gamma$ and $P \longrightarrow \gamma \gamma^{*}$ strongly depend on the value of the decay constant $f_{P}$. A brief discussion of the first-order corrections to $f_{P}$ is therefore unavoidable. Writing the result in terms of

$$
\mu_{P}=\frac{m_{P}^{2}}{16 \pi^{2} f^{2}} \ln \frac{m_{P}^{2}}{\mu^{2}}
$$

which appears in the loop calculations, one has analysis in Ref. 7. Experimentally, one has ${ }^{8}$

$$
\begin{aligned}
& \Gamma\left(\pi^{0} \rightarrow \gamma \gamma\right)=7.6 \pm 0.3 \mathrm{eV}, \\
& \Gamma(\eta \rightarrow \gamma \gamma)=0.41 \pm 0.07 \mathrm{keV}, \\
& \Gamma\left(\eta^{\prime} \rightarrow \gamma \gamma\right)=4.47 \pm 0.39 \mathrm{keV},
\end{aligned}
$$

while the decay amplitudes and widths are given by

$$
\begin{aligned}
& A(P \rightarrow \gamma \gamma)=-\frac{\sqrt{2} C_{P} \alpha}{\pi f_{P}} \epsilon^{\mu v \alpha \beta} \epsilon_{\mu} k_{\nu} \epsilon_{\alpha}^{\prime} k_{\beta}^{\prime}, \\
& \Gamma(P \rightarrow \gamma \gamma)=\frac{\alpha^{2} C_{P}^{2} m_{P}^{3}}{32 \pi^{3} f_{P}^{2}},
\end{aligned}
$$

with $C_{\pi}=1, C_{\eta_{8}}=1 / \sqrt{3}$, and $C_{\eta_{1}}=2 \sqrt{2} / \sqrt{3}$ and $k^{2}=k^{\prime 2}$ $=0$ for real photons of polarizations $\epsilon$ and $\epsilon^{\prime}$. To lowest order, one has $f_{P}=f=134 \mathrm{MeV}$ thus obtaining the satisfactory prediction $\Gamma\left(\pi^{0} \rightarrow \gamma \gamma\right)=7.8 \mathrm{eV}$. Next order corrections can arise from loops and counterterms originated by the anomalous Lagrangian (3), from the nonanomalous $f_{P}$ corrections displayed in Eqs. (5), and from the $\eta-\eta^{\prime}$ mixing phenomena. Loop corrections are found to be

$$
\begin{aligned}
& A\left(\pi^{0} \rightarrow \gamma \gamma\right)=A\left(\pi^{0} \rightarrow \gamma \gamma\right)_{\mathrm{tree}}\left[1+\left(-\frac{8}{3}+4+\frac{2}{3}-2\right) \mu_{\pi}+\left(-\frac{10}{3}+4+\frac{1}{3}-1\right) \mu_{K}\right], \\
& A\left(\eta_{8} \rightarrow \gamma \gamma\right)=A\left(\eta_{8} \rightarrow \gamma \gamma\right)_{\mathrm{tree}}\left[1+(-4+4+0+0) \mu_{\pi}+(-2+4+1-3) \mu_{K}\right], \\
& A\left(\eta_{1} \rightarrow \gamma \gamma\right)=A\left(\eta_{1} \rightarrow \gamma \gamma\right)_{\mathrm{tree}}\left[1+(-2+2+0+0) \mu_{\pi}+(-2+2+0+0) \mu_{K}\right],
\end{aligned}
$$

where the first and second terms of the chiral logarithms $\mu_{\pi, K}$ represent the contributions of $\pi^{+}, K^{+}$loops in Figs. 1 (a) and $1(\mathrm{~b})$, respectively, while the third and fourth terms are similarly due to wave-function and $f_{P}$ renormalizations. We agree with Ref. 7 on the globally vanishing $\pi$ and $K$ loop corrections, although we disagree on the independent contributions. Introducing $\eta-\eta^{\prime}$ mixing in the usual way, i.e., with

$$
\eta=\eta_{8} \cos \theta-\eta_{1} \sin \theta, \quad \eta^{\prime}=\eta_{8} \sin \theta+\eta_{1} \cos \theta
$$


one can construct the following reduced ratios ${ }^{7}$ :

$$
\begin{aligned}
\rho_{n} & =\frac{3 m_{\pi}^{3} \Gamma(\eta \rightarrow \gamma \gamma)}{m_{\eta}^{3} \Gamma\left(\pi^{0} \rightarrow \gamma \gamma\right)} \\
& =\left(\frac{f_{\pi}}{f_{\eta_{8}}} \cos \theta-\sqrt{8} \frac{f_{\pi}}{f_{\eta_{1}}} \sin \theta\right)^{2}=2.4 \pm 0.4, \\
\rho_{\eta^{\prime}} & =\frac{3 m_{\pi}^{3} \Gamma\left(\eta^{\prime} \rightarrow \gamma \gamma\right)}{8 m_{\eta^{3}}^{3} \Gamma\left(\pi^{0} \rightarrow \gamma \gamma\right)} \\
& =\left(\frac{f_{\pi}}{f_{\eta_{1}}} \cos \theta+\frac{1}{\sqrt{8}} \frac{f_{\pi}}{f_{\eta_{8}}} \sin \theta\right)^{2}=0.62 \pm 0.05,
\end{aligned}
$$

where the numerical values are the experimental results ${ }^{8}$ deduced from Eqs. (6). Taking $\theta=-19.5^{\circ} \quad(\sin \theta$ $=-\frac{1}{3}$ ), as follows from the theoretical predictions and phenomenological analyses, ${ }^{2,7,9,10}$ one can deduce $f_{\eta_{1}} / f_{\pi}$ from the experimental value of $\rho_{\eta^{\prime}}$. The obtained result is $f_{\eta_{1}} / f_{\pi}=1.1$, thus leading to the prediction $\rho_{\eta}=2.6$, in good agreement with the experimental result (10).

This numerical discussion indicates that $\mathcal{L}_{6}^{\text {anom }}$ counterterms do not play a relevant roll in $P \rightarrow \gamma \gamma$ decays. On the one hand, divergent ( $\mu$ dependent) counterterms

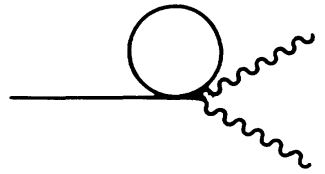

(a)

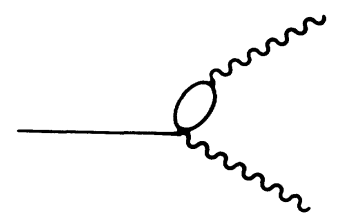

(b)

cannot be present in $\mathcal{L}_{6}^{\text {anom }}$ and, on the other hand, finite counterterms should have low-energy constants compatible with zero because of the just obtained reasonable agreement between the theoretical prediction and experimental data for $P \rightarrow \gamma \gamma$ decays.

Examples of finite counterterms contributing to $\mathcal{L}_{6}^{\text {anom }}$ are

$$
\mathcal{L}_{6}^{\mathrm{anom}}=i \epsilon^{\mu v \alpha \beta} F_{\mu \nu} F_{\alpha \beta}\left\{a_{1} \operatorname{tr}\left[m Q^{2}\left(\Sigma-\Sigma^{\dagger}\right)\right]+a_{1}^{\prime} \operatorname{tr}\left[Q^{2}\right] \operatorname{tr}\left[m \Sigma-m \Sigma^{\dagger}\right]+a_{2} \operatorname{tr}\left[m \Sigma Q \Sigma^{\dagger} Q \Sigma-m \Sigma^{\dagger} Q \Sigma Q \Sigma^{\dagger}\right]+\cdots\right\} .
$$

Notice, in particular, that (apart from the preceding discussion about the smallness of $a_{1}, a_{2}$ ) counterterms contributing to $\pi^{0} \rightarrow \gamma \gamma$ are proportional to $m_{u, d}$ or $m_{\pi}^{2}$ and as a consequence fully negligible.

A drastic change in the situation occurs when turning $P \rightarrow \gamma \gamma$ to $P \rightarrow \gamma \gamma^{*}$ decays. This case is the relevant one in $P \rightarrow \gamma l^{+} l^{-}\left(k^{2}>0\right)$ and in two-photon formation processes $\gamma \gamma^{*} \rightarrow P\left(k^{2}<0\right)$. Data on the $k^{2}$ dependence in $\pi^{0} \rightarrow \gamma e^{+} e^{-}, \eta \rightarrow \gamma \mu^{+} \mu^{-}$, or in $\gamma \gamma^{*} \rightarrow \pi^{0}, \eta$ and $\eta^{\prime}$ are (or are expected to be) available even if their quality at the moment is rather poor. The $k^{2}$ dependence, up to first-order corrections, is exclusively generated by the $\pi^{+}$and $K^{+}$ loops in Fig. 1 (b). Therefore the expression for $A\left(P \rightarrow \gamma \gamma^{*}\right)$ is given by

$$
A\left(P \rightarrow \gamma \gamma^{*}\right)=-\frac{\sqrt{2} C_{P} \alpha}{\pi f_{P}} \epsilon^{\mu \nu \alpha \beta} \epsilon_{\mu} k_{\nu} \epsilon_{\alpha}^{\prime} k_{\beta}^{\prime}\left(1+A_{P} \frac{m_{\pi}^{2}}{16 \pi^{2} f^{2}} F\left(m_{\pi}^{2}, k^{2}\right)+A_{P} \frac{m_{K}^{2}}{16 \pi^{2} f^{2}} F\left(m_{K}^{2}, k^{2}\right)\right),
$$

with $A_{P}=2$ for $P=\pi^{0}$ and $\eta_{8}$ and $A_{P}=1$ for $P=\eta_{1}$ and $F\left(m_{P}^{2}, k^{2}\right)$ is defined by

$$
F\left(m_{P}^{2}, k^{2}\right)=-\frac{x}{6} \ln \frac{m_{P}^{2}}{\mu^{2}}+\left(\frac{2}{3}-\frac{x}{6}\right)\left(\frac{x-4}{x}\right)^{1 / 2} \ln \frac{\sqrt{x}+(x-4)^{1 / 2}}{-\sqrt{x}+(x-4)^{1 / 2}}-\frac{4}{3},
$$

where $x=k^{2} / m_{P}^{2}$. Here one clearly observes the cancellation of chiral logarithms as $k^{2}=0$ (as before) and the noncancellation in the terms proportional to $k^{2}$. This latter fact implies unambiguously the existence of counterterms proportional to $k^{2}$ :

$$
\begin{aligned}
\mathcal{L}_{6}^{\mathrm{anom}}=i \epsilon^{\mu v \alpha \beta} F_{\alpha \beta} F_{v \lambda}\left\{A_{1} \operatorname{tr}\left[Q^{2} \Sigma^{\dagger} \partial_{\mu} \partial^{\lambda} \Sigma-Q^{2} \Sigma \partial_{\mu} \partial^{\lambda} \Sigma^{\dagger}-Q^{2}\left(\partial_{\mu} \partial^{\lambda} \Sigma^{\dagger}\right) \Sigma+Q^{2}\left(\partial_{\mu} \partial^{\lambda} \Sigma\right) \Sigma^{\dagger}\right]\right. \\
+A_{2} \operatorname{tr}\left[Q \Sigma^{\dagger} Q \partial_{\mu} \partial^{\lambda} \Sigma-Q \Sigma Q \partial_{\mu} \partial^{\lambda} \Sigma^{\dagger}\right]+A_{1}^{\prime} \operatorname{tr}\left(Q^{2}\right) \operatorname{tr}\left[\Sigma^{\dagger} \partial_{\mu} \partial^{\lambda} \Sigma-\Sigma \partial_{\mu} \partial^{\lambda} \Sigma^{\dagger}\right] \\
\left.+A_{2}^{\prime} \operatorname{tr}\left[Q \Sigma Q \Sigma^{\dagger}\right] \operatorname{tr}\left[\Sigma^{\dagger} \partial_{\mu} \partial^{\lambda} \Sigma-\Sigma \partial_{\mu} \partial^{\lambda} \Sigma^{\dagger}\right]\right\}+i \epsilon^{\mu v \alpha \beta} \partial^{\lambda} F_{\lambda \nu} F_{\alpha \beta} \\
\quad \times\left\{B_{1} \operatorname{tr}\left[Q^{2} \Sigma^{\dagger} \partial_{\mu} \Sigma-Q^{2} \Sigma \partial_{\mu} \Sigma^{\dagger}\right]+B_{2} \operatorname{tr}\left[Q \Sigma^{\dagger} Q \partial_{\mu} \Sigma-Q \Sigma Q \partial_{\mu} \Sigma^{\dagger}\right]\right. \\
\left.+B_{1}^{\prime} \operatorname{tr}\left(Q^{2}\right) \operatorname{tr}\left[\Sigma^{\dagger} \partial_{\mu} \Sigma\right]+B_{2}^{\prime} \operatorname{tr}\left[Q \Sigma Q \Sigma^{\dagger}\right] \operatorname{tr}\left[\Sigma^{\dagger} \partial_{\mu} \Sigma\right]\right\}+\cdots,
\end{aligned}
$$


where the counterterms containing $A_{i}$ or $B_{l}$ give contributions to $\pi^{0}, \eta_{8}$, and $\eta_{1}$, proportional to the lowest-order amplitude coming from Eq. (3) times $k^{2}$. The terms containing $A_{l}^{\prime}$ or $B_{i}^{\prime}$ contribute only to $\eta_{1} \rightarrow \gamma \gamma^{*}$. This can be rather easily seen since (suppressing derivatives) there are only two types of terms contributing to $P \rightarrow \gamma \gamma^{*}$. Namely,

$$
\operatorname{tr}\left(Q^{2} M\right), \operatorname{tr} Q^{2} \operatorname{tr} M
$$

The consequence of this is that the slopes at small $k^{2}$ are the same for $\pi^{0}$ and $\eta_{8}$, while we cannot say anything about the slope for the $\eta_{1}$.

Experimentally, nothing is known about the slope for the $\eta^{\prime}$ and the results for the $\pi^{0}$ are contradictory. ${ }^{11} \mathrm{~A}$ single experiment for the $\eta$ gives a measured slope in the decay $\eta \rightarrow \gamma \mu^{+} \mu^{-}$of $1.9 \pm 0.4 \mathrm{GeV}^{-2}$. The predictions for the slopes in quark models and vector-meson dominance have been analyzed in Ref. 12. They predict values slightly dependent on the $s$-quark content of the meson involved, namely, $1.7 \mathrm{GeV}^{-2}$ for the $\pi^{0}$ and, with $\theta=-19.5^{\circ}, 1.9 \mathrm{GeV}^{-2}$ for the $\eta$ and $1.4 \mathrm{GeV}^{-2}$ for the $\eta^{\prime}$. The loop contributions in Eq. (12) amount to about one-third of the observed value for the $\eta(1.9 \pm 0.4$ $\mathrm{GeV}^{-2}$ ). The rest should come from the counterterms (14). In any case, since the physical $\eta$ is mainly $\eta_{8}$ the slopes for $\pi^{0}$ and $\eta$ should be very similar.

The branching ratio for the $\pi^{0}$ decay,

$$
\frac{\Gamma\left(\pi^{0} \rightarrow \gamma e^{+} e^{-}\right)}{\Gamma\left(\pi^{0} \rightarrow \gamma \gamma\right)}=1.2 \%,
$$

is clearly reproduced in our context but it is not a specific test of the $k^{2}$ behavior. Just pure QED effects in the photon propagator and the $\gamma e^{+} e^{-}$vertex explain the ratio above.

In conclusion, the effects of the next-to-leading-order contributions to the two-photon decays of the pseudoscalar mesons are rather different when the two photons are on mass shell than when one of the photons is allowed to be off mass shell. In the first case the loop contributions cancel in such a way that their only effect is the U(3) breaking in the decay constants. Using the $\eta-\eta^{\prime}$ mixing angle $\theta=-19.5^{\circ}$, the obtained theoretical values for the decay widths are in good agreement with the experimental results. Therefore, the contribution of the terms from the dimension-six anomalous Lagrangian is expected to be very small. In the second case, i.e., the decays $P \rightarrow \gamma l^{+} l^{-}$and $\gamma \gamma^{*} \rightarrow P$ transitions, the terms proportional to the invariant mass of the lepton pair, $k^{2}$, are divergent. These divergences can be absorbed in the

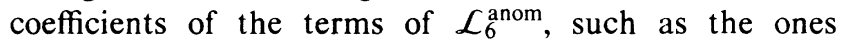
shown in Eq. (14). The dimension-six terms proportional to $k^{2}$ can be classified in two groups: terms contributing with the same weight to $\pi^{0}$ and $\eta_{8}$ decays, relative to the lowest order, and terms that only contribute to $\eta_{1}$ decays. Since the slope at $k^{2}=0$ obtained from the loop contributions is the same as for $\pi^{0}$ and $\eta_{8}$, one can conclude that this result still holds when the complete firstorder corrections are taken into account.

${ }^{1}$ R. Dashen, Phys. Rev. 185, 1248 (1969); R.Dashen and M. Weinstein, Phys. Rev. 185, 1291 (1969).

${ }^{2}$ J. Gasser and H. Leutwyler, Nucl. Phys. B250, 465,517,530 (1985), and Ann. Phys. (N.Y.) 158, 142 (1984).

${ }^{3}$ J. Wess and B. Zumino, Phys. Lett. 37B, 98 (1971).

${ }^{4}$ E. Witten, Nucl. Phys. B223, 422 (1983).

${ }^{5} \mathrm{H}$. Georgi, Weak Interactions and Modern Particle Theory (Benjamin, New York, 1984).

${ }^{6}$ H. Leutwyler and M. Roos, Z. Phys. C 25, 91 (1984).

${ }^{7}$ J. F. Donoghue et al., Phys. Rev. Lett. 59, 2766 (1985).

${ }^{8}$ M. Aguilar-Benitez (Particle Data Group), Phys. Lett. 170B, 1 (1986).

${ }^{9}$ A. Bramon, Phys. Lett. 51B, 87 (1974).

${ }^{10}$ F. J. Gilman and R. Kauffman, Phys. Rev. D 36, 2761 (1987).

${ }^{11}$ A. Bramon and E. Masso, Phys. Lett. 104B, 311 (1981).

${ }^{12}$ R. I. Djhelyadin et al., Phys. Lett. 94B, 548 (1980). 